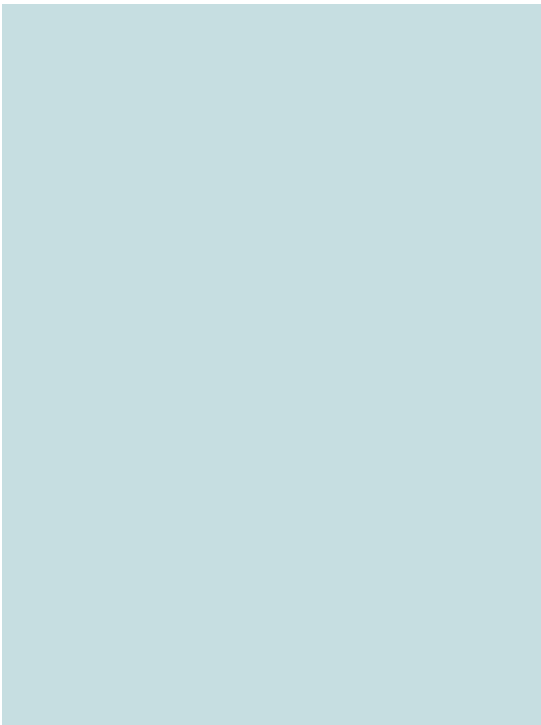

Rolf Symons, MD

Pier Giorgio Masci, MD

Kaatje Goetschalckx, MD

Kostas Doulaptsis, MD, PhD

Stefan Janssens, MD, PhD

Jan Bogaert, MD, PhD

\title{
Effect of Infarct Severity on Regional and Global Left Ventricular Remodeling in Patients with Successfully Reperfused ST Segment Elevation Myocardial Infarction ${ }^{1}$
}

Purpose:

\section{Materials and Methods:}

Results:

Conclusion:
To evaluate the relationship between myocardial infarction (MI) severity at magnetic resonance (MR) imaging and regional and global postinfarction left ventricular (LV) remodeling.

This HIPAA-compliant study was institutional review board approved. In 186 patients, reperfused ST segment elevation MI (mean age \pm standard deviation, 59 years \pm 11 ) was prospectively studied the first week and 4 months after infarction. Microvascular obstruction (MVO) and intramyocardial hemorrhage (IMH) helped define three infarct severity groups: S0, no MVO or IMH $(n=68)$; S1, MVO, no IMH $(n=84)$; and S2, MVO and IMH $(n=34)$. Results were compared in 40 control patients (mean age, 58 years \pm 10 ). One-way analysis of variance or Kruskal-Wallis test with post hoc Bonferroni correction was used. Follow-up analysis was performed with paired Student $t$ test or Mann-Whitney $U$ test.

Infarct severity was positively related $(P<.001)$ to peak of troponin I, inflammatory biomarkers, area at risk, and infarct volume and inversely related to myocardial salvage ratio, systolic wall thickening (SWT) in the infarct, and adjacent myocardium and LV ejection fraction (EF). At follow-up, LV EF significantly improved in S0 and S1 (S0: $53 \% \pm 8$ to $56 \% \pm$ $8, P<.001 ; \mathrm{S} 1: 48 \% \pm 8$ to $52 \% \pm 10, P=.006)$, while S2 adversely remodeled with increase in $\mathrm{LV}$ end-diastolic (175 $\mathrm{mL} \pm 35$ to $201 \mathrm{~mL} \pm 40)$ and end-systolic $(100 \mathrm{~mL} \pm 24$ to $115 \mathrm{~mL} \pm 29)$ volumes $(P<.001)$. SWT recovery in the infarct (S0: $32 \% \pm 21$ to $42 \% \pm 24, P<.001$; S1: $19 \% \pm 13$ to $29 \% \pm 19, P<.001 ; \mathrm{S} 2: 11 \% \pm 9$ to $15 \% \pm 15, P=.22)$ and adjacent (S0: $41 \% \pm 19$ to $52 \% \pm 21, P<.001$; S1: $32 \%$ \pm 11 to $38 \% \pm 16, P=.002 ; \mathrm{S} 2: 24 \% \pm 13$ to $29 \% \pm 14, P$ $=.092)$ and remote $(\mathrm{S} 0: 54 \% \pm 18$ to $62 \% \pm 20, P=.002$; S1: $53 \% \pm 18$ to $57 \% \pm 20, P=.092 ; \mathrm{S} 2: 50 \% \pm 35$ to $53 \%$ $\pm 22, P=.75)$ myocardium was related to infarct severity. $\mathrm{LV}$ wall thinning with $\mathrm{LV}$ mass decrease occurred at follow-up (S0: $110 \mathrm{~g} \pm 27$ to $100 \mathrm{~g} \pm 27, P<.001 ; \mathrm{S} 1: 115 \mathrm{~g} \pm 24$ to $109 \mathrm{~g} \pm 26, P=.019$; S2: $134 \mathrm{~g} \pm 35$ to $117 \mathrm{~g} \pm 27, P=.043)$.

${ }^{1}$ From the Departments of Radiology (R.S., K.D., J.B.) and Cardiovascular Diseases (K.G., S.J.), Gasthuisberg University Hospital, Herestraat 49, Leuven BR 3000, Belgium; and Department of Cardiology, Fondazione CNR/Regione Toscana G. Monasterio, Pisa, Italy (P.G.M.). Received November 29, 2013; revision requested January 7, 2014; revision received March 24; accepted May 2; final version accepted July 14 . Address correspondence to J.B. (e-mail: Jan.bogaert@uzleuven.be).
MVO and IMH significantly affect postinfarct myocardial and LV remodeling; hemorrhagic infarcts behave worse than nonhemorrhagic infarcts, with lack of functional recovery and adverse $L V$ remodeling extending to remote myocardium.

(C) RSNA, 2014

Online supplemental material is available for this article. 
E arly reperfusion therapy is the current standard of treatment in $\mathrm{pa}^{-}$ tients with acute ST segment elevation myocardial infarction (MI) (1). At the time of reperfusion, however, substantial myocardial necrosis has often occurred, and the process of irreversible myocardial damage is not necessarily ended by the restoration of the epicardial coronary flow. Reperfusion itself can induce cardiomyocyte death (ie, myocardial reperfusion injury). Moreover, many patients show microvascular obstruction (MVO) in the infarct region, caused by structural damage to the capillary bed, embolization of debris during the percutaneous coronary intervention (PCI) procedure, and the release of vasoconstrictor, thrombogenic, and inflammatory substances (2-6). Moreover, in areas with severe MVO, extravasation of

\section{Advances in Knowledge}

- The presence of microvascular obstruction and/or intramural hemorrhage in patients with reperfused ST segment elevation myocardial infarction (MI) is associated with a greater degree of irreversible myocardial damage, as evidenced by cardiac biomarkers (troponin I, S0: $31 \mu \mathrm{g} / \mathrm{L}$ [range, $13-60 \mu \mathrm{g} / \mathrm{L}$ ]; S1: $86 \mu \mathrm{g} / \mathrm{L}$ [range, $52-151 \mu \mathrm{g} / \mathrm{L}]$; and S2: $191 \mu \mathrm{g} / \mathrm{L}$ [137-268 $\mu \mathrm{g} / \mathrm{L}] ; \mathrm{P}<$ .001 ) and infarct volume (S0: 11 $\mathrm{mL} \pm 11 ; \mathrm{S} 1: 23 \mathrm{~mL} \pm 14$; and S2: $40 \mathrm{~mL} \pm 19 ; \mathrm{P}<.001)$ mea- $^{-}$ sured with late gadolinium-based contrast material-enhanced MR imaging.

- Increasing infarct severity, as evidenced by the presence of microvascular obstruction and/or intramural hemorrhage, is associated with reduced global and regional LV function early postinfarction.

- Increasing infarct severity, as evidenced by the presence of microvascular obstruction and/or intramural hemorrhage, influences postinfarction regional and global LV functional recovery and LV remodeling. blood causes an intramyocardial hemorrhage (IMH) $(7,8)$. Though myocardial infarct size is a major determinant of adverse left ventricular (LV) remodeling and worse outcome, several studies have shown that MVO and IMH portend a worse prognosis (3-13).

Cardiovascular magnetic resonance (MR) imaging has become the modality of choice to study MI at different stages postinfarction (14). It allows for a comprehensive assessment of the reversible and irreversible tissue damage and a study of the effect on regional and global LV geometry and function $(6,7,15,16)$. As it is obvious that MVO and IMH adversely affect postinfarction global LV remodeling, we hypothesized that increasing infarct severity (as gauged with MVO and IMH) could have an effect on the noninfarcted myocardium, which in turn yields global LV dilatation and dysfunction. The objective of the present study was, therefore, to evaluate the relationship between infarct severity, as determined with MR imaging, and regional and global postinfarction LV remodeling.

\section{Materials and Methods}

\section{Patient Population}

This prospective longitudinal study was undertaken in a single tertiary center.

\section{Implications for Patient Care}

- Comprehensive cardiac MR imaging in acute myocardial infarction provides important information with regard to infarct severity, allowing identification of patients with increased risk of adverse LV remodeling postinfarction.

- The effect of myocardial necrosis in reperfused ST segment elevation MI is not limited to the infarct region but significantly affects the noninfarcted myocardium, as well.

- Persistent remote myocardial dysfunction at 4-month follow-up may be an early sign of evolution toward ischemic heart failure.
Patients with acute ST segment elevation MI were prospectively enrolled between December 2007 and July 2012. Patients were included if they were older than 18 years, had cumulative ST segment elevation of $6 \mathrm{~mm}$ or more, and were successfully treated with PCI within 12 hours after symptom onset and evidence of significant LV dysfunction (dyskinesia or akinesia involving three contiguous segments or more at echocardiography performed before MR imaging by independent experts). Exclusion criteria included previous MI or coronary revascularization, pulmonary edema, cardiogenic shock, cardiomyopathy, estimated glomerular filtration rate less than $30 \mathrm{~mL} / \mathrm{min}$ per $1.73 \mathrm{~m}^{2}$, major comorbidities that limit life expectancy, or contraindications to MR imaging. No standard protocol was used for troponin and creatine kinase MB collection. Patients were studied in the first week and 4 months after the event. PCI and cardiac rehabilitation were performed according to guideline-based local practice, with early use of concomitant antiplatelet

Published online before print 10.1148/radiol.14132746 Content codes: CA MR

Radiology 2015; 274:93-102

\section{Abbreviations:}

$\mathrm{AAR}=$ area at risk

$\mathrm{EDV}=$ end-diastolic volume

$\mathrm{EF}=$ ejection fraction

$\mathrm{ESV}=$ end-systolic volume

$\mathrm{IMH}=$ intramyocardial hemorrhage

LGE = late gadolinium-based contrast material

enhancement

$\mathrm{LV}=$ left ventricular

$\mathrm{MI}=$ myocardial infarction

$\mathrm{MVO}=$ microvascular obstruction

$\mathrm{PCl}=$ percutaneous coronary intervention

SWT = systolic wall thickening

\section{Author contributions:}

Guarantors of integrity of entire study, R.S., K.D., J.B. study concepts/study design or data acquisition or data analysis/interpretation, all authors; manuscript drafting or manuscript revision for important intellectual content, all authors; approval of final version of submitted manuscript all authors; literature research, R.S., P.G.M., K.G., K.D., J.B.; clinical studies, all authors; experimental studies, P.G.M., K.D.; statistical analysis, R.S., P.G.M., K.D., J.B.; and manuscript editing, R.S., P.G.M., K.G., K.D., J.B.

Conflicts of interest are listed at the end of this article. 
and anticoagulant medications. To better appraise the magnitude and importance of observed functional and morphologic changes, patients with MI were compared with an age- and sexmatched control group of patients with no evidence of coronary artery disease and absent late gadolinium-based contrast material enhancement (LGE) on MR images. Because of the exploratory nature of this study, no sample size calculations were made prior to the onset of the study. Written informed consent was obtained for all subjects, and the study was approved by the ethics review board of the University Hospital Leuven, Leuven, Belgium.

\section{MR Imaging Protocol}

Image acquisition was performed with a 1.5-T system (Intera; Philips Medical Systems, Best, the Netherlands) by using commercially available cardiac MR software, electrocardiographic triggering, and a cardiac-dedicated phase-array coil. After determination of cardiac axes with localizers, LV function was assessed by using breath-hold steadystate free precession cine MR imaging (repetition time msec/echo time msec, 3.6/1.8; flip angle, $60^{\circ}$; section thickness, $8 \mathrm{~mm}$; matrix, $160 \times 256$; field of view, $300 \mathrm{~mm}$; pixel size, $1.6 \times$ $1.6 \mathrm{~mm}$; and number of phases, 30). In cardiac short-axis direction, LV was completely encompassed by contiguous sections. Next, T2-weighted MR imaging was performed in the cardiac short-axis direction by using a darkblood T2-weighted short-tau inversion-recovery fast spin-echo sequence (repetition time, two heart beats; echo time, 100 msec; inversion time, 180 msec; turbo factor, 33; matrix, 160 $\times 256$; field of view, $350 \mathrm{~mm}$; section thickness, $8 \mathrm{~mm}$; 8-10 contiguous sections) to assess the area at risk (AAR) and IMH. A breath-hold, T1-weighted, three-dimensional contrast materialenhanced inversion-recovery gradientecho sequence (4.5/1.3; flip angle, $15^{\circ}$; 20 contiguous sections; section thickness, $5 \mathrm{~mm}$; matrix, $128 \times 256$; field of view, $350 \mathrm{~mm}$; pixel size, $1.4 \times 1.4$ $\mathrm{mm})$ was used to depict the presence of MVO and MI. An intravenous contrast material dose of $0.2 \mathrm{mmol}$ of gadopentetate dimeglumine per kilogram of body weight was used. Presence and extent of MVO were evaluated in early imaging - that is, within the first 5 minutes after contrast material administration-while LGE imaging (after 10-25 minutes) was used to evaluate MI extent. Inversion time was individually adapted to suppress the signal of normal myocardial tissue.

Forty age- and sex-matched subjects (mean age \pm standard deviation, 58 years $\pm 10 ; 34$ men [85\%] and six women [15\%]) with clinical suspicion of coronary artery disease, in whom significant coronary artery disease was ruled out by means of fractional flow reserve measurement at invasive coronary angiography, were prospectively recruited at our center to undergo a similar MR imaging protocol as discussed earlier, including a first-pass stress perfusion sequence. Perfusion imaging consisted of three short-axis sections acquired every heartbeat. Rest imaging preceded stress by at least 10 minutes. Imaging parameters were a balanced turbo gradient-echo sequence with $3 / 1.5$; flip angle, $50^{\circ}$; $90^{\circ}$ prepulse; $100-\mathrm{msec}$ prepulse delay; section thickness, $10 \mathrm{~mm}$; and pixel size, $2.7 \times 2.9 \mathrm{~mm}$. For stress imaging, $140 \mu \mathrm{g} / \mathrm{kg} / \mathrm{min}$ of adenosine was administered intravenously for approximately 4 minutes. Imaging commenced 3 minutes after starting the infusion and continued until the end of the stress perfusion acquisition. For each perfusion study, a dose of $0.05 \mathrm{mmol} / \mathrm{kg}$ gadopentetate dimeglumine was used. An additional dose of $0.1 \mathrm{mmol} / \mathrm{kg}$ was administered for LGE imaging. The total administered dose in control patients and infarct patients was similar (ie, 0.2 $\mathrm{mmol} / \mathrm{kg}$ ). To be considered as a control subject, first-pass perfusion findings had to be negative, with no evidence of myocardial enhancement at LGE imaging.

\section{Image Analysis}

For all quantitative analyses, dedicated MR evaluation software was used (ViewForum; Philips Medical Systems, Best, the Netherlands). Image analysis was performed by a single observer (J.B.) with more than 10 years of experience in cardiovascular MR imaging. For evaluation of global LV function and myocardial mass, endocardial and epicardial borders were manually traced in end-diastolic and end-systolic short-axis sections. End diastole and end systole were defined as the largest and smallest LV cavity, respectively, determined at the midventricular short-axis level. Papillary muscles and trabeculations were not included in the myocardium. End-systolic volumes were corrected for longitudinal shortening, excluding atrially located short-axis sections at end systole from analysis. Summation of delineated slides yielded LV end-diastolic volume (EDV) and end-systolic volume (ESV). LV ejection fraction (EF) was determined as the difference between LV EDV and LV ESV, divided by LV EDV and expressed as a percentage. Myocardial mass was obtained by multiplying myocardial volume by the specific density of myocardial tissue (ie, $1.05 \mathrm{~g} /$ $\mathrm{mL}$ ). To quantify regional myocardial morphology and function, a three-compartment approach (infarct, adjacent, remote) was performed by merging cine and LGE images, as described more extensively elsewhere $(6,16)$. The location and extent of late myocardial enhancement was used to define the infarcted myocardium on the corresponding short-axis cine MR images. The adjacent periinfarct territory was defined by using an arbitrary angle of $30^{\circ}$ on both sides of the infarcted myocardium on all sections, showing late myocardial enhancement (Fig 1). In the longitudinal ventricular direction on the first section of nonenhanced myocardium, the myocardium in immediate contact with the enhanced myocardium was considered adjacent, as well, while the remaining myocardium was considered remote. By merging LGE and cine images, a single value of end-diastolic and endsystolic wall thickness was obtained for all three compartments. Systolic wall thickening (SWT) was determined as the difference between end-systolic and end-diastolic wall thickness, divided by end-diastolic wall thickness and expressed as a percentage. Negative SWT values indicate compartments with systolic wall thinning. 


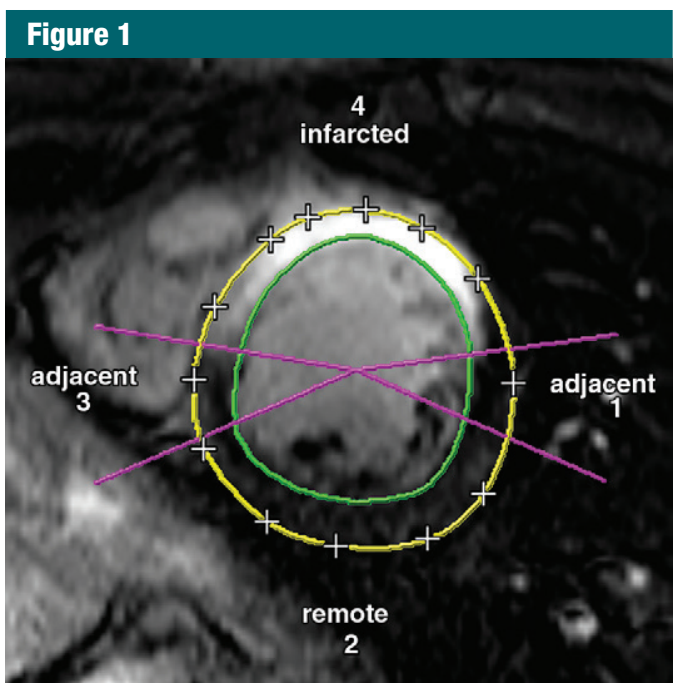

Figure 1: Midventricular short-axis LGE MR image (4.3/1.3, 280-msec inversion time) obtained 12 minutes after contrast material administration illustrates the three-compartment approach to divide the myocardium into an infarcted region, two adjacent (covering an angle of $30^{\circ}$ ) regions, and a remote region.

MI volume was determined on LGE images by using the extent of enhanced myocardium. MVO volume was determined in early postcontrast imaging by using the hypointense zone in the infarct-related myocardium. Both were measured by manually tracing the suspected area on each short-axis section. When present, the zone of MVO was included in infarct volume estimation. Infarct transmurality was calculated as the ratio of mean thickness of enhanced myocardium to mean thickness of the corresponding myocardial wall. A signal intensity value of the myocardium supplied by the infarct-related artery 2 standard deviations above the signal intensity of remote myocardium was considered the AAR. IMH was defined as a hypointense area in the center of the AAR with a signal intensity 2 standard deviations below the signal intensity of the periphery of the AAR, having a minimal volume of $1 \mathrm{~mL}\left(1 \mathrm{~cm}^{3}\right)$ (7). For the AAR calculations, the hemorrhagic area was included in the AAR. Myocardial salvage ratio was determined as infarct size at baseline, divided by the AAR at baseline and expressed as a percentage. Follow-up variation $(\Delta)$ of LV ESV was determined as the difference in LV ESV between follow-up and baseline, divided by LV ESV at baseline and expressed as a percentage. An $\Delta \mathrm{LV}$ ESV percentage of at least $15 \%$ was considered to indicate adverse LV remodeling at follow-up (17). Three groups of infarct severity were defined: Patients with no MVO or IMH were labeled S0, patients with MVO but without IMH were labeled S1, and patients with both MVO and IMH were labeled S2.

\section{Statistical Analysis}

Data were tested for normal distribution by using the Shapiro-Wilk test. Continuous normally distributed data were expressed as means \pm standard deviations. Nonparametric data were expressed as median values with interquartile ranges. Categorical variables were expressed as frequency with percentage. For the infarct severity groups, continuous variables were compared by using the one-way analysis of variance or KruskalWallis test, as appropriate. When the results indicated presence of significant differences between groups, we made post hoc pairwise group comparisons by using the Student $t$ test or Mann-Whitney $U$ test as appropriate, with Bonferroni correction. A comparison between categorical variables was performed by using the $\chi^{2}$ test or Fisher exact test, as appropriate. The Student paired $t$ test or Wilcoxon test was used as appropriate to compare continuous variable differences between baseline and follow-up. A Spearman rank order correlation was run to determine the relationship between changes in LV ESV and SWT in the infarcted myocardium. The distribution of SWT in the infarct region at 4 months was tested by means of onesample Kolmogorov-Smirnov analysis. Univariate linear regression analysis was performed to assess the variables associated with SWT in the infarct region at 4 months. Clinical and regionalbased variables with a significance level of $P<.10$ at univariate analysis were then introduced as covariates in the multivariate linear regression analysis to assess the independent determinants of SWT in the infarct region at follow-up. A two-sided $P$ value of less than .05 was considered to indicate a significant difference. All analyses were performed with SPSS version 17.0 software (SPSS, Chicago, Ill).

\section{Results}

\section{Patient Characteristics}

Of 200 eligible patients, 14 patients were excluded because of refusal to participate $(n=3)$, loss during follow-up $(n=4)$, or insufficient MR image quality $(n=7)$. The remaining 186 patients underwent MR imaging within 1 week (median, 70 hours; interquartile range, 47-96 hours) and 4 months after the acute event. During follow-up, six patients were readmitted because of recurrent angina (of which four underwent PCI for restenosis), and five patients were hospitalized because of heart failure (four in the S0 group, four in the $\mathrm{S} 1$ group, and three in the $\mathrm{S} 2$ group; $P=.702$ ). No cardiac deaths or reinfarction occurred during the first 4 months postinfarction. By using the MR-based infarct severity classification, 68 patients $(36 \%)$ had no evidence of MVO or IMH (S0), 84 patients (45\%) had MVO without IMH (S1), and 34 patients $(18 \%)$ had MVO and IMH 
Figure 2
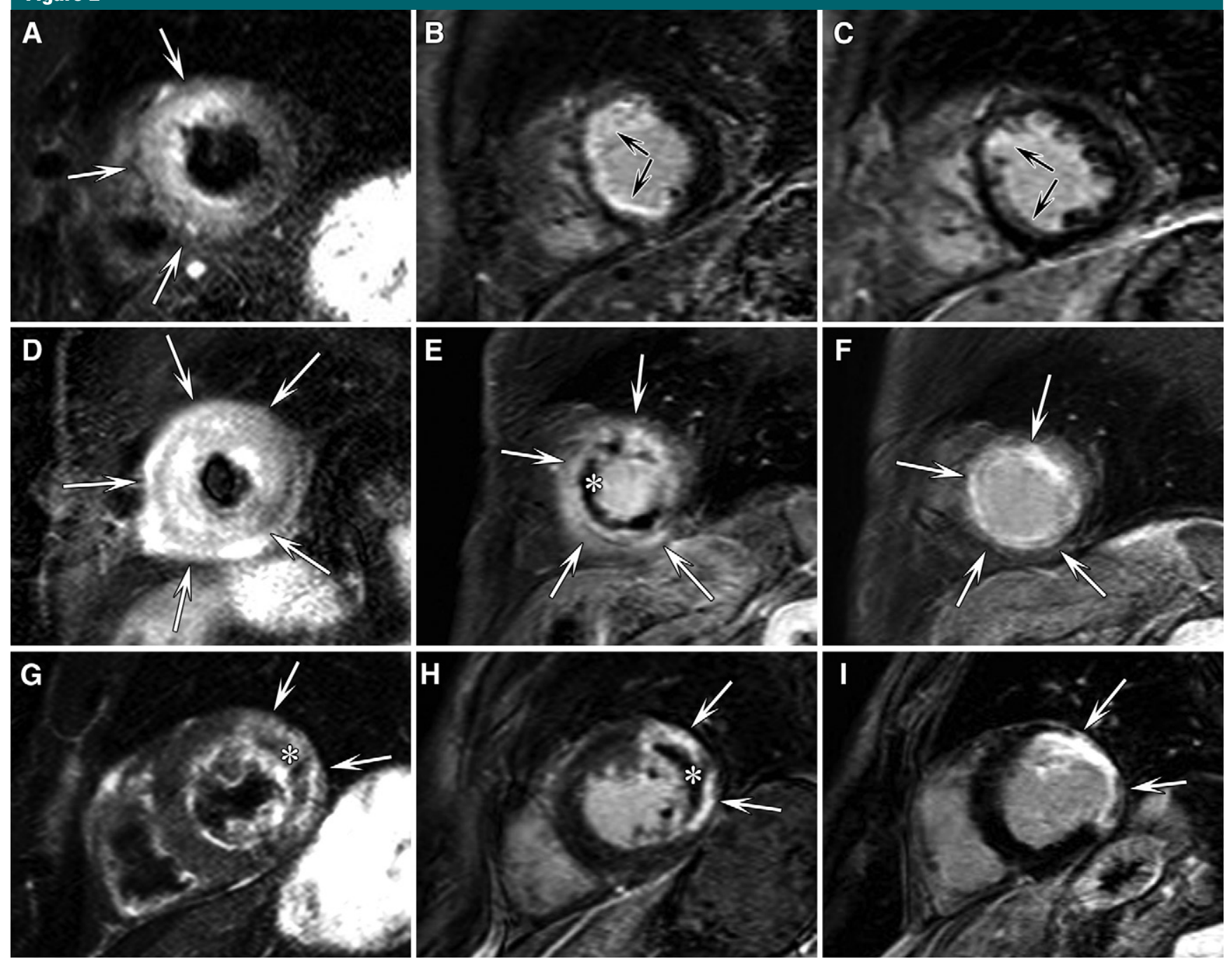

Figure 2: Cardiac short-axis MR images demonstrate increasing infarct severity. $A, D, G$, Short-axis T2-weighted short-tau inversion-recovery fast spin-echo images (two heart beats per $100 \mathrm{msec}, 180$-msec inversion time, $1.4 \times 2.2-\mathrm{mm}$ pixel size) were acquired. Gadolinium-based contrast material-enhanced MR images were obtained 15 minutes after contrast material administration (4.3/1.3) $B, E, H$, in the first week after infarction and $C, F$, I, at 4 months after infarction. The inversion time was individually adjusted to suppress noninfarcted myocardium. $A, B, C$, The patient classified as SO shows myocardial edema in the apical anteroseptal LV wall (arrows on $A$ ) with $50 \%$ transmural enhancement after contrast material administration (arrows on $B$ ). At 4-month follow-up, the initial infarct volume decreased from $16.7 \mathrm{~mL}$ to $8.6 \mathrm{~mL}$ (arrows on $C$ ). D, E, F, The patient classified as $S 1$ shows diffuse edema of the LV apex (arrows on $D$ ). There is transmural enhancement (arrows on $E$ ) with a central zone of microvascular obstruction (* on $E$ ). There was a decrease in infarct volume at 4-month follow-up, from $34.6 \mathrm{~mL}$ to $20.1 \mathrm{~mL}$ (arrows on $F$ ). G, H, I, The patient classified as S2 shows myocardial edema in the lateral LV wall (arrows on $G$ ) with a large central hypointense area, reflecting intramyocardial hemorrhage (* on $G$ ). After contrast material administration, transmural myocardial enhancement is present (arrows on $H$ ) with large microvascular obstruction (* on H). At follow-up, important thinning of the infarcted myocardium is found (arrows on /), with decrease of the infarct volume from $66.5 \mathrm{~mL}$ to $33.1 \mathrm{~mL}$.

(S2) (Fig 2). No significant differences in baseline variables were seen among the groups or with the control group (Table 1). Peak troponin I and creatine kinase $\mathrm{MB}$ values increased significantly (both $P<.001$ ) (Table 1) with increasing infarct severity. No significant differences in time from symptom onset to reperfusion or in culprit coronary artery distribution pattern were found between groups (Table 1).

\section{Morphology and Function at Baseline}

Increasing infarct severity was associated with significantly larger AAR, greater infarct volume, and infarct transmurality but conversely with lower myocardial salvage ratios (all $P<.001$ ) (Tables 2, 3). The relative number of transmural infarcts increased with increasing infarct severity-that is, $18 \%$ in S0, $24 \%$ in S1, and $65 \%$ in S2. When compared with end-diastolic wall thickness in control patients, patients with 


\section{Table 1}

\section{Patient Characteristics}

\begin{tabular}{|c|c|c|c|c|c|c|c|}
\hline \multirow[b]{2}{*}{ Parameter } & \multicolumn{7}{|l|}{ Control } \\
\hline & Patients $(n=40)$ & Patients $(n=186)$ & $P$ Value & So Group $(n=68)$ & S1 Group $(n=84)$ & S2 Group $(n=34)$ & $P$ Value \\
\hline $\operatorname{Age}(y)^{*}$ & $58 \pm 10$ & $59 \pm 12$ & $.66^{\dagger}$ & $59 \pm 11$ & $59 \pm 12$ & $59 \pm 12$ & $.99^{\ddagger}$ \\
\hline No. of men & $34(85)$ & $155(83)$ & $.79^{\S}$ & $51(75)$ & $72(86)$ & $32(94)^{\|}$ & $.037^{\S}$ \\
\hline No. of patients with arterial hypertension & $16(40)$ & $60(32)$ & $.48^{\S}$ & $24(35)$ & $22(26)$ & $14(41)$ & $.23^{\S}$ \\
\hline Body surface area $\left(m^{2}\right)^{\star}$ & $1.92 \pm 0.17$ & $1.92 \pm 0.20$ & $.99^{\dagger}$ & $1.90 \pm 0.22$ & $1.94 \pm 0.20$ & $1.94 \pm 0.16$ & $.43^{\ddagger}$ \\
\hline No. of patients with anterior MI & NA & $84(45)$ & NA & $30(44)$ & $38(45)$ & $16(47)$ & $.96^{\S}$ \\
\hline $\begin{array}{l}\text { Systolic blood pressure at } \\
\text { admission }(\mathrm{mm} \mathrm{Hg})^{\star}\end{array}$ & $130 \pm 23$ & $132 \pm 22$ & $.59^{\dagger}$ & $131 \pm 24$ & $133 \pm 21$ & $132 \pm 24$ & $.79^{\ddagger}$ \\
\hline $\begin{array}{l}\text { Diastolic blood pressure at } \\
\text { admission }(\mathrm{mm} \mathrm{Hg})^{\star}\end{array}$ & $74 \pm 13$ & $77 \pm 13$ & $.26^{\dagger}$ & $76 \pm 14$ & $77 \pm 13$ & $77 \pm 13$ & $.73^{\ddagger}$ \\
\hline Peak creatine kinase MB level (U/L) ${ }^{\#}$ & NA & $212(117-394)$ & NA & $120(47-250)$ & $215(153-407)^{\|}$ & $384(275-501)^{\| \star \star}$ & $<.001^{\dagger+}$ \\
\hline Peak troponin I level $(\mu \mathrm{g} / \mathrm{L})^{\#}$ & NA & $76(31-147)$ & NA & $31(13-60)$ & $86(52-151)^{\|}$ & $191(137-268)^{\| \star \star}$ & $<.001^{t+}$ \\
\hline \multicolumn{8}{|l|}{ Culprit coronary artery } \\
\hline Left anterior descending coronary artery & NA & $86(46.2)$ & NA & $31(46)$ & $40(48)$ & $15(44)$ & $.93^{\S}$ \\
\hline Right coronary artery & NA & $83(44.6)$ & NA & $33(48)$ & $37(44)$ & $13(38)$ & $.61^{\S}$ \\
\hline Left circumflex coronary artery & NA & $17(9.1)$ & NA & $4(6)$ & $7(8)$ & $6(18)$ & $.14^{\ddagger}$ \\
\hline Time to $\mathrm{PCl}(\mathrm{min})^{\#}$ & NA & $210(150-318)$ & NA & $205(150-304)$ & $217(148-308)$ & $215(169-377)$ & $.91^{\S}$ \\
\hline Time between $\mathrm{PCl}$ and MR imaging (h)\# & NA & $70(47-96)$ & NA & $71(48-96)$ & $71(48-96)$ & $62(46-77)$ & $.30^{\S}$ \\
\hline \multicolumn{8}{|l|}{$\begin{array}{l}\text { No. of patients with medical treatment } \\
\text { at discharge }\end{array}$} \\
\hline $\begin{array}{l}\text { Angiotensin-converting enzyme inhibitors } \\
\text { and/or angiotensin receptor blockers }\end{array}$ & $4(10)$ & $172(92)$ & $<.001^{\#}$ & $60(88)$ & $80(95)$ & $32(94)$ & $.25^{\S}$ \\
\hline Beta-blockers & $5(12)$ & $157(84)$ & $<.001^{\#}$ & $56(82)$ & $73(87)$ & $28(82)$ & $.70^{\S}$ \\
\hline Statins & $7(18)$ & $172(92)$ & $<.001^{\ddagger}$ & $63(93)$ & $78(93)$ & $31(91)$ & $.95^{\S}$ \\
\hline Antiplatelet agents & $2(5)$ & $178(96)$ & $<.001^{\ddagger \ddagger}$ & $65(96)$ & $81(96)$ & $32(94)$ & $.85^{\S}$ \\
\hline Diuretics & 1 (2) & $135(72)$ & $<.001^{\#}$ & $50(74)$ & $59(70)$ & $26(76)$ & $.77^{\S}$ \\
\hline
\end{tabular}

Note.-Except where indicated, data are numbers of patients, with percentages in parentheses. To convert from units per liter to microkatals per liter, multiply by 0.0167 . NA $=$ not applicable.

* Data are means \pm standard deviations, unless indicated otherwise.

${ }^{\dagger}$ Calculated with the Student $t$ test.

₹ Calculated with one-way analysis of variance.

$\S$ Calculated with the $\chi^{2}$ test.

"Bonferroni-adjusted $P<.016$ versus S0 group.

\# Unless otherwise indicated, data are medians, with interquartile ranges in parentheses.

** Bonferroni-adjusted $P<.016$ versus the $\mathrm{S} 1$ group.

tt Calculated with the Kruskal-Wallis test.

\# Calculated with the Fisher exact test.

infarct showed significantly higher end-diastolic wall thickness values not only in the infarcted region but also in the noninfarcted adjacent and remote myocardium $(P<.001)$ (Table E1 [online]). Conversely, SWT in patients with infarct was significantly less than in control patients $(<.001$ for all comparisons with control patients) (Fig 3). The impairment in SWT was most pronounced in the infarcted region, but, to a lesser extent, was also detected in the adjacent and remote regions $(P<$ .001 for all comparisons with control patients) (Fig 3). The severity of impairment in SWT was positively related to infarct severity in the infarcted and adjacent myocardium $(P<.001$ for the difference between severity groups) (Fig 3). With increasing infarct severity, $L V$ EDV $(P=.007), \operatorname{LV} \operatorname{ESV}(P$ $<.001)$, and LV mass $(P<.001)$ increased, while LV EF $(P<.001)$ decreased (Table 2).

\section{Evolution of LV Morphology and Function}

At follow-up, LV EF improved significantly in the S0 and S1 groups (both $P$
$<.001$ ), but not in the S2 group (Table $2)$. On the contrary, the S2 group showed adverse LV remodeling with a significant increase in both LV EDV and LV ESV (both $P<.001$ ), an increase not present in the $\mathrm{S} 0$ and $\mathrm{S} 1$ groups (Table 2) (Fig 2). All groups showed a decrease in LV mass and a wall thinning at follow-up $(P<.001)$ (Table E1 [online]). The latter was not limited to the infarct region but involved the adjacent and, to a lesser extent, the remote region, as well. In the infarct region, the magnitude of wall thinning 


\section{Table 2}

\section{Volumes, Mass, and Function in Patients and Control Patients}

\begin{tabular}{|c|c|c|c|c|c|c|c|}
\hline Parameter & $\begin{array}{l}\text { Control } \\
\text { Patients }\end{array}$ & Patients & $P$ Value & So Group & S1 Group & S2 Group & $P$ Value \\
\hline \multicolumn{8}{|l|}{ LV EDV (mL) } \\
\hline 1 week & $162 \pm 13$ & $158 \pm 34$ & $.18^{*}$ & $148 \pm 32$ & $158 \pm 33$ & $175 \pm 35^{\dagger \ddagger}$ & $<.001^{\S}$ \\
\hline 4 months & $\ldots$ & $167 \pm 41$ & $.17^{\star}$ & $153 \pm 32$ & $166 \pm 40$ & $201 \pm 40^{\dagger \ddagger}$ & $<.001^{\S}$ \\
\hline$P$ value & $\ldots$ & $<.001^{\|}$ & $\ldots$ & $.26^{\|}$ & $.038^{\|}$ & $<.001^{\|}$ & $\ldots$ \\
\hline \multicolumn{8}{|l|}{ LV ESV (mL) } \\
\hline 1 week & $64 \pm 10$ & $81 \pm 25$ & $<.001^{\star}$ & $71 \pm 22$ & $82 \pm 23^{\dagger}$ & $100 \pm 24^{\dagger \ddagger}$ & $<.001^{\S}$ \\
\hline 4 months & $\ldots$ & $82 \pm 30$ & $<.001^{\star}$ & $67 \pm 23$ & $80 \pm 25^{\dagger}$ & $115 \pm 29^{\dagger \ddagger}$ & $<.001^{\S}$ \\
\hline$P$ value & $\ldots$ & $.96^{\|}$ & $\ldots$ & $.15^{\|}$ & $.35^{\|}$ & $.001^{\|}$ & $\ldots$ \\
\hline \multicolumn{8}{|l|}{ LV EF (\%) } \\
\hline 1 week & $60 \pm 6$ & $49 \pm 9$ & $<.001^{*}$ & $53 \pm 8$ & $48 \pm 8^{\dagger}$ & $43 \pm 8^{\dagger \ddagger}$ & $<.001^{\S}$ \\
\hline 4 months & $\ldots$ & $52 \pm 10$ & .030 & $56 \pm 8$ & $52 \pm 10^{\dagger}$ & $43 \pm 8^{\dagger \ddagger}$ & $<.001^{\S}$ \\
\hline$P$ value & $\ldots$ & $<.001^{\|}$ & $\ldots$ & $<.001^{\prime \prime}$ & $.006^{\|}$ & $.51^{\|}$ & $\ldots$ \\
\hline \multicolumn{8}{|l|}{ LV mass $(\mathrm{g})$} \\
\hline 1 week & $99 \pm 24$ & $117 \pm 28$ & $<.001^{*}$ & $110 \pm 27$ & $115 \pm 24$ & $134 \pm 35^{\dagger \ddagger}$ & $<.001^{\S}$ \\
\hline 4 months & $\ldots$ & $107 \pm 27$ & $.09^{\star}$ & $100 \pm 27$ & $109 \pm 26$ & $117 \pm 27^{\dagger}$ & $.009^{\S}$ \\
\hline$P$ value & $\ldots$ & $<.001^{\|}$ & $\ldots$ & $<.001^{\|}$ & $.019^{\|}$ & $.043^{\|}$ & $\ldots$ \\
\hline $\begin{array}{l}\text { Percentage } \Delta L V \\
\qquad E S V \geq 15 \%^{\#}\end{array}$ & NA & $44(25)$ & NA & $10(15)$ & $18(23)$ & $16(50)^{\dagger \ddagger}$ & $<.001^{\star \star}$ \\
\hline
\end{tabular}

Note.-Except where indicated, data are means \pm standard deviations. NA $=$ not applicable.

* Calculated with the Student $t$ test.

${ }^{\dagger}$ Bonferroni-adjusted $P<.016$ versus the S0 group.

‡ Bonferroni-adjusted $P<.016$ versus the $\mathrm{S} 1$ group.

$\S$ Calculated with one-way analysis of variance.

" Calculated with the Student paired $t$ test.

\# Data are numbers of patients, with percentages in parentheses.

${ }^{\star \star}$ Calculated with the $\chi^{2}$ test.

\section{Table 3}

\section{Infarct Characteristics at Baseline and Follow-up}

\begin{tabular}{lccccc} 
Parameter & Mean Value & S0 Group & S1 Group & S2 Group & $P$ Value \\
\hline $\begin{array}{l}\text { Area at risk }(\mathrm{mL}) \\
\quad 1 \text { week }\end{array}$ & $38 \pm 22$ & $26 \pm 20$ & $40 \pm 18^{\star}$ & $56 \pm 21^{\star \dagger}$ & $<.001^{\ddagger}$ \\
Infarct volume $(\mathrm{mL})$ & & & & \\
$\quad 1$ week & $22 \pm 17$ & $11 \pm 11$ & $23 \pm 14^{\star}$ & $40 \pm 19^{\star \dagger}$ & $<.001^{\ddagger}$ \\
$\quad 4$ months & $11 \pm 9$ & $6 \pm 7$ & $12 \pm 8^{\star}$ & $21 \pm 9^{\star \dagger}$ & $<.001^{\ddagger}$ \\
$\quad P$ value & $<.001^{\S}$ & $<.001^{\S}$ & $<.001^{\S}$ & $<.001^{\S}$ & \\
Infarct volume/area at risk (\%) & & & & & \\
$\quad 1$ week & $57 \pm 26$ & $42 \pm 23$ & $61 \pm 25^{\star}$ & $75 \pm 20^{\star \dagger}$ & $<.001^{\ddagger}$ \\
Infarct transmurality (\%) & & & & & \\
$\quad \begin{array}{l}1 \text { week } \\
4 \text { months }\end{array}$ & $77 \pm 18$ & $63 \pm 15$ & $82 \pm 13^{\star}$ & $92 \pm 12^{\star \dagger}$ & $<.001^{\ddagger}$ \\
$P$ value & $68 \pm 19$ & $52 \pm 12$ & $73 \pm 15^{\star}$ & $87 \pm 12^{\star \dagger}$ & $<.001^{\ddagger}$ \\
& $<.001^{\S}$ & $<.001^{\S}$ & $<.001^{\S}$ & $.002^{\S}$ & $<.001^{\ddagger}$
\end{tabular}

Note.-Unless indicated otherwise, data are means \pm standard deviations.

* Bonferroni-adjusted $P<.016$ versus the S0 group.

${ }^{\dagger}$ Bonferroni-adjusted $P<.016$ versus the $\mathrm{S} 1$ group.

$\ddagger$ Calculated with one-way analysis of variance.

$\S$ Calculated with the Student paired $t$ test. was positively related to infarct severity. Partial functional recovery in SWT was present in the infarcted and adjacent myocardium in the S0 and S1 groups (both $P<.01$ ), but not in the $\mathrm{S} 2$ group $(P=.22)$ (Fig 3$)$. In the remote myocardium, functional recovery was found in the S0 group $(P=.002)$, a trend toward recovery was present in the S1 group $(P=.09)$, and the S2 group did not show improvement $(P=$ .70) (Fig 3). Adverse LV remodeling occurred more frequently in the S2 group $(P<.001)$ (Table 2). Changes in LV ESV were inversely related to recovery in SWT $(r=-0.284 ; 95 \%$ confidence interval: $-0.415,-0.141)$.

\section{Determinants of SWT in Infarcted Myocardium at Follow-up}

The Shapiro-Wilk test for normality showed a normal distribution for SWT in the infarct region at follow-up $(P=$ .39). At univariate linear regression analysis, male sex, greater end-diastolic wall thickness in the infarct region, infarct transmurality, and higher infarct severity at baseline were associated with lower SWT in the infarcted myocardium at follow-up. On the other hand, a positive relationship was observed between SWT of the infarct region at baseline and follow-up (Table E2 [online]). Multivariate linear regression analysis showed that higher infarct severity remained a significant predictor of lower SWT in the infarcted myocardium at follow-up, even after correction for the other covariates, including infarct transmurality and SWT at baseline (Table E3 [online]).

\section{Discussion}

In this study, we used comprehensive MR imaging to evaluate the effect of MVO and IMH on LV remodeling in the first 4 months postinfarction in 186 patients who underwent successful reperfusion of ST segment elevation MI. The major finding of our study is that infarct severity as assessed with cardiovascular MR imaging within 1 week of an ST segment elevation MI is associated with larger infarctions and is an important predictor of adverse LV remodeling 


\section{Figure 3}

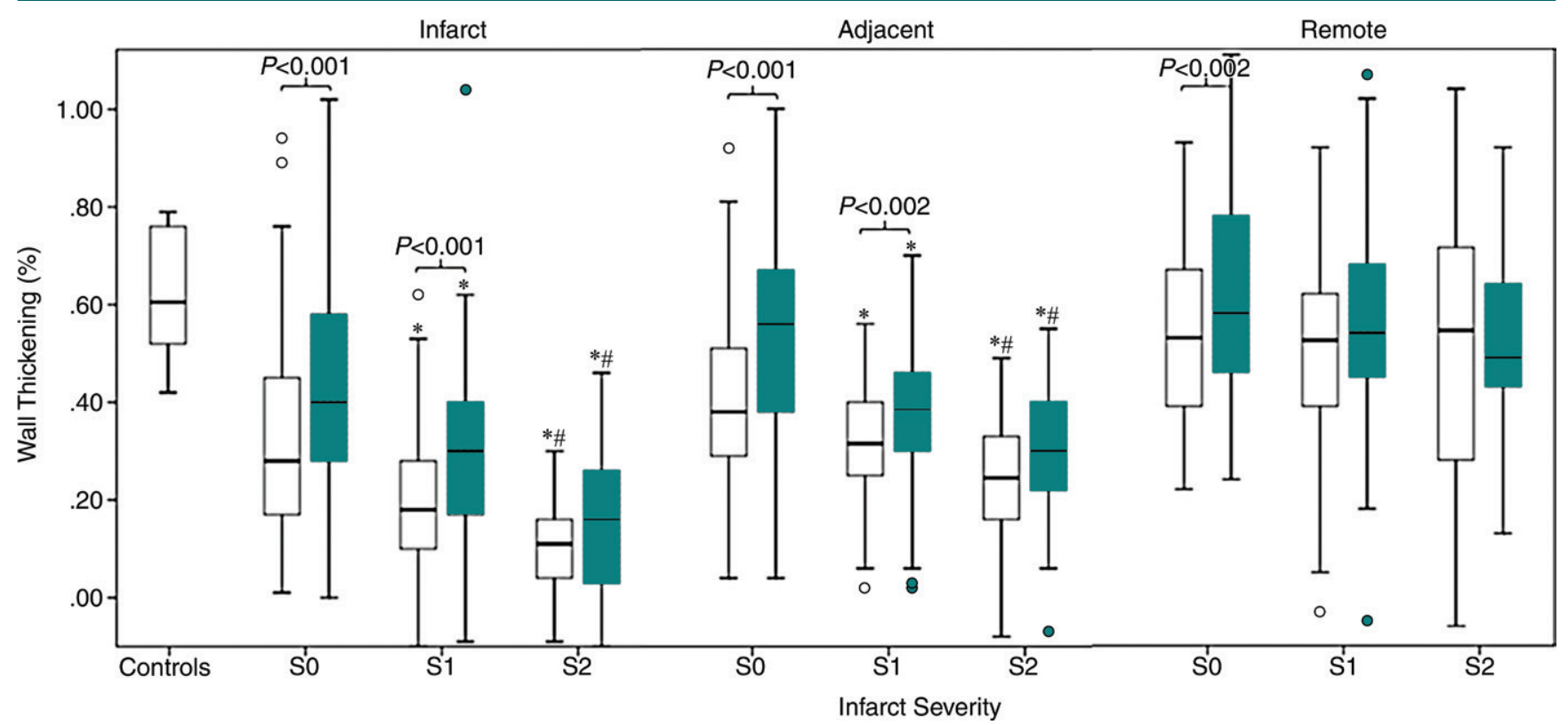

Figure 3: Box plots show systolic wall thickening in control patients and in the infarcted, adjacent, and remote myocardium in the different infarct severity groups at 1 week (white boxes) and 4 months (blue boxes) of follow-up. $P$ values between infarct severity groups were calculated with one-way analysis of variance and the Student $t$ test, as appropriate. $P<.001$ for the differences between severity groups in infarcted and adjacent myocardium. $*=$ Bonferroni-adjusted $P<.016$ versus the S0 group, \# = Bonferroni-adjusted $P<.016$ versus the S1 group. $P$ values between 1 week and 4 months were calculated by using the Student paired $t$ test. White and blue circles represent outliers.

after 4 months. In addition, this LV remodeling process involves not only the infarcted myocardium, but also the adjacent and remote myocardium.

\section{Infarct Severity and LV Function}

Our study results are consistent with previous data, showing that the presence of MVO, and, more importantly, IMH, is related to more extensive infarcts, as evidenced by cardiac biomarkers, AAR, and LGE burden (ie, infarct size and transmurality) $(7,9,11)$. Since the distribution pattern of the culprit coronary artery and the time to reperfusion were similar between infarct severity groups, it can be questioned whether the phenomenon of MVO and IMH itself contributes to a larger $\mathrm{AAR}$ and infarct volume. Moreover, myocardial salvage is inversely related to infarct severity (from nearly $60 \%$ in S0 to $25 \%$ in S2), emphasizing limited salvageable myocardium in hemorrhagic infarcts. Early postinfarction, LV EF and SWT are inversely related to infarct severity. Patients with a hemorrhagic infarct show significantly larger LV EDV, not only in comparison to control patients but also with respect to S0 and S1 groups. This finding suggests an early onset of adverse LV remodeling in the hemorrhagic group. The effect of infarct severity on SWT early postinfarction is most obvious in the infarcted and adjacent myocardium. In the infarcted myocardium, this can be attributed to a greater infarct transmurality with increasing infarct severity and to structural myocardial damage not limited to the myocytes but affecting the microcirculation (in case of MVO), with additional interstitial extravasation of erythrocytes (in case of IMH). In the adjacent myocardium, altered loading conditions and mechanical tethering of viable myocardium adjacent to necrotic myocardium are likely mechanisms and can explain why the dysfunction increases with increasing infarct severity $(18,19)$. However, as we used LGE imaging to define the infarct region, stunned myocardium in the adjacent region may be another mechanism that causes dysfunction soon after infarction in the adjacent region.
As such, it can be hypothesized that in less severe infarcts that show a higher myocardial salvage index, myocardial stunning prevails (or at least contributes to a larger extent), while in severe infarcts, tethering is the principal mechanism of periinfarct dysfunction (20). Additionally, this hypothesis could explain the divergence in functional recovery in the adjacent myocardium at follow-up, depending on infarct severity (see the following section).

In all patients with infarct, remote SWT was significantly impaired when compared with control patients, emphasizing that the noninfarcted myocardium contributes to the decrease in LV EF. This finding is concordant with previous MR imaging studies (with or without MR tagging), showing remote myocardial dysfunction due to altered coronary vasodilatory properties, changes in regional mechanical load, and mechanical tethering to infarcted regions $(18,21-23)$.

Patients with infarct show, in comparison to control patients, a significantly 
higher wall thickness, inclusive of the noninfarcted remote myocardium. Whether this is a preexisting condition (eg, due to unrecognized arterial hypertension in the infarct group) or reflects a so-called early LV remodeling due to an inflammatory response with interstitial fibrosis and edema, is unclear at the moment $(24,25)$. Moreover, in the infarcted myocardium, infarct severity is associated with increase in LV wall thickness. This can be explained by more extensive myocardial damage, with increased cellular swelling and interstitial edema in severe infarcts.

\section{Infarct Severity and LV Remodeling}

LV remodeling in the first months after infarction is significantly determined by infarct severity. Nonhemorrhagic infarcts improve LV function, while hemorrhagic infarcts do not recover functionally, but instead show adverse $\mathrm{LV}$ remodeling with significant increases in LV EDV and LV ESV at follow-up. At the myocardial level, a similar phenomenon is observed with partial functional recovery in the infarcted and adjacent myocardium in the nonhemorrhagic infarcts, which is not present in the hemorrhagic group. This divergence in functional recovery can be extended toward the remote myocardium, with significant functional recovery in the S0 group, a trend toward functional recovery in the $\mathrm{S} 1$ group, and lack of functional recovery in the $\mathrm{S} 2$ group. Although the exact explanation for the latter phenomenon remains speculative (eg, inflammatory-mediated, reshaping of the left ventricle), it suggests that persistent remote myocardial dysfunction may be important in the evolution toward ischemic dilated cardiomyopathy. Structurally, a wall thinning is found at follow-up that is most pronounced in the infarcted myocardium. The magnitude of wall thinning is the largest in hemorrhagic infarcts, which can be attributed to the more extensive myocardial damage (eg, greater infarct transmurality) being replaced by a thinner fibrotic scar $(25,26)$.

\section{Limitations}

Some study limitations should be mentioned. First, we do not have preinfarct data with regard to arterial blood pressures. This hampers a correct interpretation with regard to the nature of the increased (remote) wall thickness at baseline (preexisting hypertrophy as opposed to infarct-related remodeling). Second, we used dark-blood T2-weighted fast spin-echo sequences to assess IMH. Other study findings suggested that $\mathrm{T} 2 *$-weighted sequences may be more sensitive in detecting IMH than T2-weighted sequences $(27,28)$. Third, although we found LV remodeling to be related to infarct severity, this was not associated with increased mortality at 4-month follow-up. Longer follow-up is needed to evaluate whether patients with increased infarct severity have worse outcome, although the MR findings may lead to a more intensive antiremodeling therapy in patients with a severe infarct. Fourth, although, theoretically, the lack of improvement in SWT at follow-up in the S2 group could be related to the smaller sample size in this group, the regional findings are nicely in line with the lack of LV EF $(P$ $=.51$ ) recovery in this group. Finally, since our study involved a single center, larger multicenter studies are needed to confirm our findings.

In conclusion, the presence of MVO and IMH has a significant effect on postinfarct myocardial and LV remodeling. In particular, hemorrhagic infarcts behave worse than nonhemorrhagic infarcts, with lack of functional recovery and adverse LV remodeling involving the infarcted and noninfarcted myocardium.

Disclosures of Conflicts of Interest: R.S. disclosed no relevant relationships. P.G.M. disclosed no relevant relationships. K.G. disclosed no relevant relationships. K.D. disclosed no relevant relationships. S.J. disclosed no relevant relationships. J.B. disclosed no relevant relationships.

\section{References}

1. Gershlick AH, Banning AP, Myat A, Verheugt FW, Gersh BJ. Reperfusion therapy for STEMI: is there still a role for thrombolysis in the era of primary percutaneous coronary intervention? Lancet 2013;382(9892):624-632.

2. Rochitte CE, Lima JA, Bluemke DA, et al. Magnitude and time course of microvascular obstruction and tissue injury after acute myocardial infarction. Circulation 1998;98(10):1006-1014.

3. Fröhlich GM, Meier P, White SK, Yellon DM, Hausenloy DJ. Myocardial reperfusion injury: looking beyond primary PCI. Eur Heart J 2013;34(23):1714-1722.

4. Ito H, Maruyama A, Iwakura K, et al. Clinical implications of the 'no reflow' phenomenon. A predictor of complications and left ventricular remodeling in reperfused anterior wall myocardial infarction. Circulation 1996;93(2):223-228.

5. Wu KC, Zerhouni EA, Judd RM, et al. Prognostic significance of microvascular obstruction by magnetic resonance imaging in patients with acute myocardial infarction. Circulation 1998;97(8):765-772.

6. Bogaert J, Kalantzi M, Rademakers FE, Dymarkowski S, Janssens S. Determinants and impact of microvascular obstruction in successfully reperfused ST-segment elevation myocardial infarction. Assessment by magnetic resonance imaging. Eur Radiol 2007;17(10):2572-2580.

7. Ganame J, Messalli G, Dymarkowski S, et al. Impact of myocardial haemorrhage on left ventricular function and remodelling in patients with reperfused acute myocardial infarction. Eur Heart J 2009;30(12):1440-1449.

8. Basso C, Corbetti F, Silva C, et al. Morphologic validation of reperfused hemorrhagic myocardial infarction by cardiovascular magnetic resonance. Am J Cardiol 2007;100(8):1322-1327.

9. Hombach V, Grebe O, Merkle N, et al. Sequelae of acute myocardial infarction regarding cardiac structure and function and their prognostic significance as assessed by magnetic resonance imaging. Eur Heart J 2005;26(6):549-557.

10. Ørn S, Manhenke C, Greve OJ, et al. Microvascular obstruction is a major determinant of infarct healing and subsequent left ventricular remodelling following primary percutaneous coronary intervention. Eur Heart J 2009;30(16):1978-1985.

11. Eitel I, Kubusch K, Strohm O, et al. Prognostic value and determinants of a hypointense infarct core in T2-weighted cardiac magnetic resonance in acute reperfused STelevation-myocardial infarction. Circ Cardiovasc Imaging 2011;4(4):354-362.

12. Mather AN, Fairbairn TA, Ball SG, Greenwood JP, Plein S. Reperfusion haemorrhage as determined by cardiovascular MRI is a predictor of adverse left ventricular remodelling and markers of late arrhythmic risk. Heart 2011;97(6):453-459. 
13. Husser O, Monmeneu JV, Sanchis J, et al. Cardiovascular magnetic resonance-derived intramyocardial hemorrhage after STEMI: influence on long-term prognosis, adverse left ventricular remodeling and relationship with microvascular obstruction. Int J Cardiol 2013;167(5):2047-2054.

14. Kim HW, Farzaneh-Far A, Kim RJ. Cardiovascular magnetic resonance in patients with myocardial infarction: current and emerging applications. J Am Coll Cardiol 2009; 55(1):1-16.

15. Dall'Armellina E, Karamitsos TD, Neubauer S, Choudhury RP. CMR for characterization of the myocardium in acute coronary syndromes. Nat Rev Cardiol 2010;7(11): 624-636.

16. Masci PG, Dymarkowski S, Rademakers FE, Bogaert J. Determination of regional ejection fraction in patients with myocardial infarction by using merged late gadolinium enhancement and cine MR: feasibility study. Radiology 2009;250(1):50-60.

17. Masci PG, Ganame J, Francone M, et al. Relationship between location and size of myocardial infarction and their reciprocal influences on post-infarction left ventricular remodelling. Eur Heart J 2011;32(13):16401648.
18. Lima JAC, Becker LC, Melin JA, et al. Impaired thickening of nonischemic myocardium during acute regional ischemia in the dog. Circulation 1985;71(5):1048-1059.

19. Mewton N, Croisille P, Revel D, Weber O, Higgins CB, Saeed M. Left ventricular postmyocardial infarction remodeling studied by combining MR-tagging with delayed MR contrast enhancement. Invest Radiol 2008;43(4): 219-228.

20. Jackson BM, Gorman JH III, Moainie SL, et al. Extension of borderzone myocardium in postinfarction dilated cardiomyopathy. J Am Coll Cardiol 2002;40(6):1160-1167; discussion 1168-1171.

21. Kramer CM, Rogers WJ, Theobald TM, Power TP, Petruolo S, Reichek N. Remote noninfarcted region dysfunction soon after first anterior myocardial infarction. A magnetic resonance tagging study. Circulation 1996;94(4):660-666.

22. Bogaert J, Bosmans H, Maes A, Suetens P, Marchal G, Rademakers FE. Remote myocardial dysfunction after acute anterior myocardial infarction: impact of left ventricular shape on regional function: a magnetic resonance myocardial tagging study. J Am Coll Cardiol 2000;35(6):1525-1534.
23. Chan W, Duffy SJ, White DA, et al. Acute lef ventricular remodeling following myocardial infarction: coupling of regional healing with remote extracellular matrix expansion. JACC Cardiovasc Imaging 2012;5(9):884-893.

24. Lee WW, Marinelli B, van der Laan AM, et al. PET/MRI of inflammation in myocardial infarction. J Am Coll Cardiol 2012;59(2): 153-163.

25. Rademakers F, Van de Werf F, Mortelmans L, Marchal G, Bogaert J. Evolution of regional performance after an acute anterior myocardial infarction in humans using magnetic resonance tagging. J Physiol 2003; 546(Pt 3):777-787.

26. McKay RG, Pfeffer MA, Pasternak RC, et al. Left ventricular remodeling after myocardial infarction: a corollary to infarct expansion. Circulation 1986;74(4):693-702.

27. O'Regan DP, Ariff B, Neuwirth C, Tan Y, Durighel G, Cook SA. Assessment of severe reperfusion injury with $\mathrm{T} 2{ }^{*}$ cardiac MRI in patients with acute myocardial infarction. Heart 2010;96(23):1885-1891.

28. Kali A, Tang RL, Kumar A, Min JK, Dharmakumar R. Detection of acute reperfusion myocardial hemorrhage with cardiac MR imaging: T2 versus T2. Radiology 2013;269(2): 387-395. 